\title{
Correction to: Comparison of Proactive Braking Intervention System Acceptability via Field Operation Tests in Different Regions
}

\author{
Takuma Ito ${ }^{1}$ - Ryosuke Matsumi ${ }^{1} \cdot$ Yuichi Saito $^{2} \cdot$ Akito Yamasaki $^{3} \cdot$ Shintaro Inoue $^{4} \cdot$ Tsukasa Shimizu $^{5}$. \\ Masao Nagai ${ }^{6} \cdot$ Hideo Inoue $^{7} \cdot$ Minoru Kamata $^{1}$
}

๑) The Author(s), under exclusive licence to Intelligent Transportation Systems Japan 2021

\section{Correction to: International Journal of Intelligent Transportation Systems Research https://doi.org/10.1007/s13177-021-00278-x}

The original article has been corrected. Due to the mistakes of the proofing team, the order of reference list was misarranged. It should be arranged as follows.

- Reference list in the first published article which contained mistakes

16. Matsumi, R., Oya, K., Ito, T., Saito, Y., Mio, M., Hashimoto, N., Nagai, M., Inoue, H., Kamata, M.: Acceptability Investigation of Proactive Braking Intervention for Elderly Drivers on Community Roads. Transactions of Society of Automotive Engineers of Japan 50(3), 911-917 (2019). (in Japanese)

17. Ito, T., Soya, M., Tohriyama, K., Saito, Y., Shimizu, T., Yamasaki, A., Nagai, M., Inoue, H.,

The original article can be found online at https://doi.org/10.1007/ s13177-021-00278-x.

Takuma Ito

ito.t@hnl.t.u-tokyo.ac.jp

1 The University of Tokyo, 7-3-1 Hongo, Bunkyo, Tokyo, Japan

2 Tokyo University of Agriculture and Technology, 2-24-16 Nakacho, Koganei, Tokyo, Japan

3 Meijo University, 1-501 Shiogamaguchi, Tenpaku, Nagoya, Aichi, Japan

4 Toyota Motor Corporation, 1200 Mishuku, Susono, Shizuoka, Japan

5 Toyota Central R\&D Labs., Inc, 41-1 Yokomichi, Nagakute, Aichi, Japan

6 Japan Automotive Research Institute, 2530 Karima, Tsukuba, Ibaraki, Japan

7 Kanagawa Institute of Technology, 1030 Shimo-ogino, Atsugi, Kanagawa, Japan
Kamata, M.: Evaluation of Acceptability of Adaptive Proactive Braking Intervention System Based on Risk Map for Elderly Drivers. International Journal of Automotive Engineering 11(2), 40-48 (2020)

18. Geospatial Information Authority of Japan: GSI Maps (online), https://maps.gsi.go.jp/, (accessed on 2020-03-22).

19. Brain Function Test Committee of Japan Society for Higher Brain Dysfunction, Trail Making Test Japanese edition (TMTJ), Shinkoh Igaku Shuppan Co. Ltd., (2019). (in Japanese)

20. Armitage, S.G.: An analysis of certain psychological tests used for the evaluation of brain injury. Psychol. Monogr. 60(1), 1-48 (1946)

21. Ito, T., Soya, M., Nakamura, S., Saito, S., Uchida, N., Kamata, M.: Acceptability of a Proactive Braking Intervention System by Elderly Drivers Using an Actual Vehicle. International Journal of Automotive Engineering 9(4), 186-194 (2018)

- Reference list in the corrected article

16. Ito, T., Soya, M., Nakamura, S., Saito, S., Uchida, N., Kamata, M.: Acceptability of a Proactive Braking Intervention System by Elderly Drivers Using an Actual Vehicle. International Journal of Automotive Engineering 9(4), 186-194 (2018)

17. Matsumi, R., Oya, K., Ito, T., Saito, Y., Mio, M., Hashimoto, N., Nagai, M., Inoue, H., Kamata, M.: Acceptability Investigation of Proactive Braking Intervention for Elderly Drivers on Community Roads. Transactions of Society of Automotive Engineers of Japan 50(3), 911-917 (2019). (in Japanese)

18. Ito, T., Soya, M., Tohriyama, K., Saito, Y., Shimizu, T., Yamasaki, A., Nagai, M., Inoue, H., Kamata, M.: Evaluation of Acceptability of Adaptive Proactive Braking Intervention System Based on Risk 
Map for Elderly Drivers. International Journal of Automotive Engineering 11(2), 40-48 (2020)

19. Geospatial Information Authority of Japan: GSI Maps (online), https://maps.gsi.go.jp/, (accessed on 2020-03-22).

20. Brain Function Test Committee of Japan Society for Higher Brain Dysfunction, Trail Making Test Japanese edition (TMTJ), Shinkoh Igaku Shuppan Co. Ltd., (2019). (in Japanese).
21. Armitage, S.G.: An analysis of certain psychological tests used for the evaluation of brain injury. Psychol. Monogr. 60(1), 1-48 (1946)

Publisher's Note Springer Nature remains neutral with regard to jurisdictional claims in published maps and institutional affiliations. 